\title{
Sharing knowledge is the key to success in a patient-physician relationship: how to produce a patient information leaflet on COPD
}

\author{
D. Scala1, S. Cozzolino1, G. D'Amato2, G. Cocco², A. Sena², \\ P. Martucci2, E. Ferraro' 1 , A.A. Mancini ${ }^{1}$
}

ABSTRACT: Sharing knowledge is the key to success in a patient-physician relationship: how to produce a patient information leaflet on COPD. D. Scala, S. Cozzolino, G. D'Amato G. Cocco, A. Sena, P. Martucci, E. Ferraro, A.A. Mancini.

Background. Chronic Obstructive Pulmonary Disease (COPD) is a leading cause of morbidity and mortality worldwide, and its prevalence is rising. In Italy, respiratory diseases are the third most common cause of death. The aim of the study is to produce a patient information leaflet (PIL) designed to educate patients about COPD in accordance with the best recommendations based on evidence and guidelines for the production of good quality written information, and to evaluate the impact of this intervention on the patients' knowledge of COPD.

Methods. The study was conducted in the Department of Chest Diseases of the Cardarelli Hospital, Naples, Italy. A total of 166 patients admitted with a diagnosis of COPD participated in the study. Patients were asked to answer 10 multiple-choice questions compiled to assess their knowledge of the disease and then to read the leaflet. Two days later they were asked to complete the questionnaire again to assess their post-intervention knowledge. Analysis of the data was performed using SPSS version 15.0.

Results. After reading the leaflet, a statistically significant increase in the proportion of correct responses was noted $(p<0.001$ by Wilcoxon signed rank test). Patients had retained the knowledge gained at the one year followup ( $p<0.05$ by Cochran's $Q$ test).

Conclusions. An educational intervention directed at adults with COPD had a positive impact on the patients' knowledge of COPD and this effect is long lasting. Monaldi Arch Chest Dis 2008; 69: 2, 50-54.

Keywords: Chronic Obstructive Pulmonary Disease, Evaluation study, Pamphlet, Patient education, Quality control.

${ }^{1}$ Biotechnology Centre, High Speciality Hospital A. Cardarelli, Naples,

2 Department of Chest Diseases, High Speciality Hospital A. Cardarelli, Naples, Italy.

Correspondence: Daniela Scala, Biotechnology Centre, High Speciality Hospital A. Cardarelli, Naples, Italy; e-mail: daniela.scala@ospedalecardarelli.it

\section{Introduction}

Chronic Obstructive Pulmonary Disease (COPD) is a degenerative disease characterized by chronic airflow obstruction due to bronchitis, emphysema, or both $[1,2]$. COPD is a leading cause of morbidity and mortality worldwide, and its prevalence is rising $[3,4,5,6]$.

In Italy respiratory diseases are the third most common cause of death. Among these, COPD represents $50-55 \%$ [7].

In the Campania Region, an area in southern Italy with 5.7 million inhabitants (10\% of the Italian population), the standard mortality rate for COPD is approximately 39.9 per 100,000 inhabitants (vs. 29.9:100.000 in the rest of Italy, calculated on the basis of ISTAT data for the year 2000). There is also a high impact of morbidity for COPD on the regional healthcare system with approxi- mately 13,000 hospital discharges (11,162 inpatients, rate 199.7/100.000 in.) in the year 2002 (crude rate 224,7/100.000 in.). The morbidity data sources are the national (www.ministerosalute.it) and regional (www.arsan.campania.it) hospital discharge registers.

For patients, impaired quality of life is often the main reason for hospital presentation and admission. Although admission offers effective treatment of acute exacerbations, management of the chronic problems of fatigue, poor exercise tolerance, and depression are often catered for inadequately. The aim of the present study is to produce a patient information leaflet (PIL) designed to educate patients about COPD in accordance with the best recommendations based on evidence and guidelines for the production of good quality written information $[8,9,10]$. A secondary objective is to evaluate the impact of this simple intervention 
on the patients' knowledge of COPD. We hypothesised that the information provided would improve the patients' knowledge about the disease.

\section{Methods}

Research on Medline for articles published between 1992 and 2003 was performed together with the retrieval of information from the web. A review of all the materials collected was performed. The leaflet content was established and preliminary drafts were evaluated and discussed with respiratory specialists. The PIL was developed according to formal guidelines for the production of good quality written information, tested for readability, Flesch-Vacca grade level 70 and Kincaid grade level 8 , and was presented in a clear format with simple language that patients found acceptable $[8,9,10,11,12]$. Ten multiple-choice questions, written at Flesch-Vacca grade level 70 and designed to take less than ten minutes to complete, were developed to assess respondents' knowledge of COPD. Questions were related to the PIL content. Patient involvement was necessary in order to assess understanding, acceptability, and content adequacy, and to clarify any ambiguities in the PIL and questionnaire [9, 13, 14]. For this purpose, a study was conducted to determine if the information provided was well communicated and satisfied people's needs. We collected suggestions from the patients and then the necessary changes were made and incorporated into the final draft of the PIL as well as the questionnaire.

The PIL was presented on a three-folded, A4 paper format, and included basic information about COPD: breathing and coughing, symptoms of exacerbation, therapeutic actions, oxygen therapy, suggestions on day-to-day and leisure activities, advice on adopting a healthy lifestyle (smoking cessation, nutrition,). The total number of the words was 601 with 4 illustrations.

Patients were asked to answer the questionnaire to determine their baseline knowledge and then to read the PIL. There was no time limit for reading the leaflet. We checked how patients complied with the reading thanks to the nursing staff who had been instructed to give patients only the routine information. Two days after, they were asked to complete the questionnaire again to assess their post-intervention knowledge. The long-term effect of the PIL was assessed one year later using the same questionnaire.

\section{Participants}

The study was conducted from 2002 to 2004 in the Chest Department of the largest specialised hospital in Southern Italy.

According to our Ethics Committee guidelines, ethics approval was not necessary for this study since it did not involve the use of drugs and/or surgical procedures. Informed consent was obtained from all patients.

Patients were eligible if they met the following conditions:
1) stable COPD (respiratory symptoms and medications unchanged for at least four weeks before the enrolment);

2) forced expiratory volume in 1 second $\left(\mathrm{FEV}_{1}\right)$ after the use of bronchodilator between $25 \%$ and $70 \%$ of the predicted normal value and the ratio of $\mathrm{FEV}_{1}$ to forced vital capacity less than $70 \%$. Patients were excluded if they had a previous diagnosis of asthma, terminal disease, dementia or uncontrolled psychiatric illness or if they had participated in a respiratory rehabilitation programme in the past year.

\section{Statistical analysis}

Analysis of the data was performed using SPSS version 15.0. The value 0 or 1 is given to each question according to how exact the answer is. Results are presented as median (25th $-75^{\text {th }}$ percentile) and interquartile range. Non parametric tests were used to compare the knowledge of participants on the pre-intervention and post-intervention phases. McNemar's test and Cochran's Q test were used to examine the changes in the number of correct and incorrect answers to the individual question. The Wilcoxon signed rank test was used to examine overall changes in the proportion of correct answers before and after the educational intervention. All tests of significance were two-sided, $p<0.05$.

\section{Results}

A total of 200 patients were approached in the pulmonary Dept. of the Cardarelli Hospital. One hundred and eighty four consented to participate in the study. Of those who refused, 4 were on hypercapnic encephalopathy and twelve claimed to be illiterate. Of the 184 who four and were eligible to participate, $18(9,8 \%)$ completed only the pre-intervention questionnaire and the remaining 166 $(90,2 \%)$ completed both questionnaires.

The patients' mean \pm S.D. age was $66.9 \pm 9,6$ years (range 39-84) and $81.9 \%$ (136) of the sample were men. Almost all were retired. 66.3\% (110) of the subjects had only a primary education, $22.9 \%$ (38) had received a secondary education, $8.4 \%$ (14) had also received a tertiary education, and $2.4 \%$ (4) had a degree.

Table 1 shows the patients' characteristics and table 2 shows simple descriptive statistics (medi-

Table 1. - Patients' Characteristics

\begin{tabular}{lcc}
\hline & N & \% \\
\hline Patient & 166 & 90.2 \\
Age & $66.9 \pm 9.6$ & - \\
Male sex & 136 & 81.9 \\
Education, n (\%) & & \\
$\quad$ Primary & 110 & 66.3 \\
$\quad$ Secondary $\quad 38$ & 22.9 \\
$\quad$ Tertiary (undergraduate) & 14 & 8.4 \\
$\quad$ University & 4 & 2.4 \\
\hline
\end{tabular}


Table 2. - Descriptives and Percentiles

\begin{tabular}{lcccc}
\hline \multicolumn{5}{c}{ Descriptive } \\
\hline & Median & Minimum & Maximum & Interquartile Range \\
\hline Pre-intervention & 6.00 & 2 & 10 & 2.00 \\
Post-intervention & 9.00 & 1 & 10 & 1.00 \\
\hline \multicolumn{5}{c}{ Percentiles } \\
\hline
\end{tabular}

ans, interquartile range) related to the pre and postintervention questionnaires.

After reading the PIL, a statistically significant increase in the proportion of correct answers was noted ( $p<0.001$ by Wilcoxon signed rank test).

The proportion of correct answers on the preintervention questionnaire varied from $40.4 \%$ (67) to questions 4 to $95,2.7 \%$ (158) to question 9. Only $3 \%$ (5) of participants gave the correct answers to all 10 questions before reading the PIL. After reading it, $24.1 \%$ (40) of participants answered all 10 questions correctly. Figure 1 shows the proportion of patients answering each question correctly before and after reading the PIL. No statistically significant association was found between the proportion of correct answers and gender, age, and educational level. The educational level didn't predict a better understanding and retention of knowledge. In fact those with a higher educational level improved their knowledge after reading the information leaflet as much as those with a lower educational level.
One year later we were able to get answers from 82 out of the 166 who joined the study. Results showed that the knowledge gained was retained after one year ( $p<0.05$ by Cochran's Q test) with only an non significant decrease in the proportion of correct answers except for the one regarding emergencies that showed further improvement (figure 2).

\section{Discussion}

PILs, such as the one evaluated in this study, are used and distributed frequently by healthcare professionals as part of educational strategies for patients.

Leaflets are seen as one of the most appropriate and least expensive means of providing patient information [12, 15]. In recent years, there has been an increase in the production of health-related written information. To be effective, PILs must be understood by patients. Unfortunately available material is often written at levels beyond the patient's literacy level $[16,17]$.

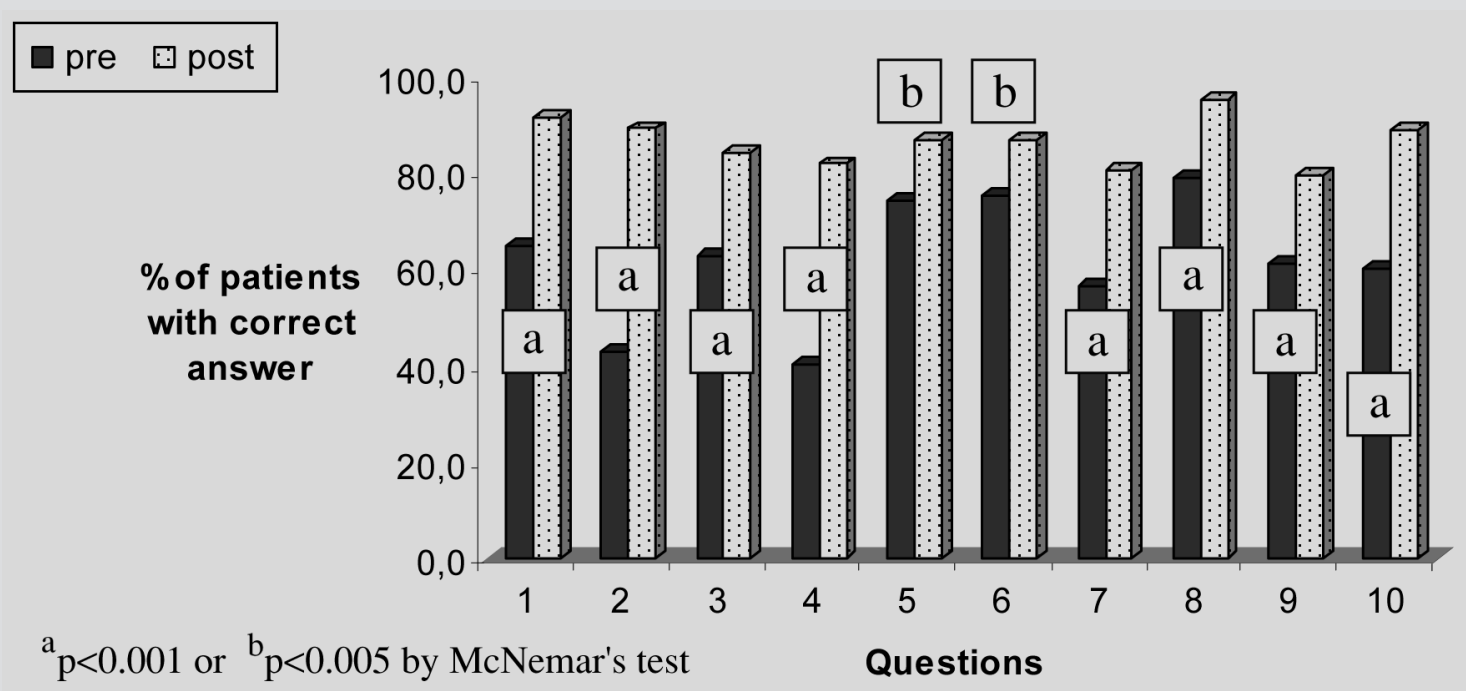

Fig. 1. - Proportion of patients answering each question correctly before and after reading the PIL. 

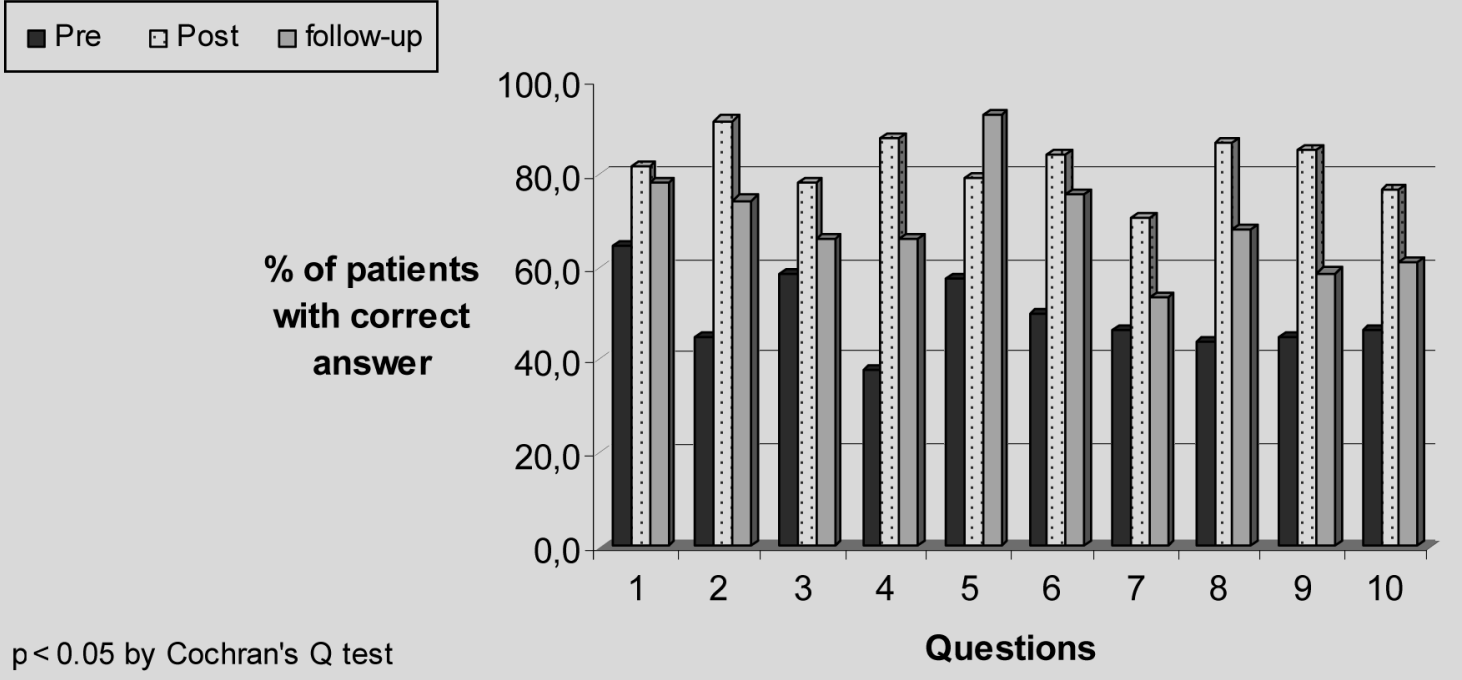

Fig. 2. - Follow-up.

Our objective was to provide COPD patients with a PIL that was actually effective in increasing their knowledge of their disease in the short and long term.

The most notable finding of this study is the marked improvement of the patients' background knowledge of COPD after reading the PIL and its persistence over time. Although some topics like the explanation of the dyspnoea, the spirometric examination, and the oxygen therapy are difficult to comprehend, the majority of patients showed a substantial gain of knowledge in these fields comparing pre and post intervention knowledge scores, suggesting that the information provided had been clearly understood by almost all study patients. In our opinion this could be the result of patient's involvement in the realisation of the PIL. The PIL, as well as the questionnaire, were piloted on a small number of patients. Patients were asked to read the PIL, to fill in the questionnaire and to highlight unclear statements. Suggestions for improvement were concerned with clinical check ups and diet that appeared to be among the least understood aspects of COPD. "Clinical check ups" is an unfamiliar phrase not widely used by our patients; this expression was changed in the PIL, as well as in the questionnaire. The majority of patients answered the question regarding the diet incorrectly because they associated the word "diet" with a low-calorie regimen. The word "diet" was replaced with the generic "food". Apart from these two points, the pilot study enabled us to incorporate minor revisions to ensure the material was understandable and in an easy-to-read format, as deemed from the patients' perspective.

Less evident improvements were seen in the two answers regarding the aetiology of COPD and the physical activity. One explanation could be the patients' belief about the aetiology of the disease and the patients' fear of physical activity. These two aspects probably need a comprehensive patient support programme, of which the educational material is a central component. One year later, knowledge improvement was still significant suggesting that the availability of the PIL always results in persistent knowledge gained over time.

One limitation of our approach was the use of a single institution and the relatively small sample size. According to international literature, patients needs vary a lot depending on the various phases of the disease and the social and cultural environment [18]. We recruited only patients admitted to our hospital, but in the future we can extend the study to other institutions. Another limitation is the fact that the PIL simply considers knowledge improvement rather than changes in behaviour as a result of the information provided with the PIL. Knowledge alone does not ensure adherence, although it is a fundamental component of any adherence-enhancement intervention [19]. The role and usefulness of alternative models in educational programmes should be explored in order to solve management problems such as poor adherence to pharmacological and non pharmacological recommendations.

In conclusion, the present study assesses the immediate and long-term impact of a PIL on the knowledge of COPD in a group of patients with COPD. Further studies should be planned to investigate broad behavioural change and should not only be aimed at improving patients' knowledge of COPD. The challenge for patient education is to achieve better strategies to address the patients' behaviour in a systematic way. This may involve a more comprehensive assessment of factors associated with patients' social and COPD management strategies. 


\section{References}

1. Mannino DM. COPD: epidemiology, prevalence, morbidity and mortality, and disease heterogeneity. Chest 2002; 121 (5 Suppl): 121S-126S.

2. de Marco R, Accordini S, Cerveri I, et al. An international survey of chronic obstructive pulmonary disease in young adults according to GOLD stages. Thorax 2004; 59: 120-5.

3. Calverley PM, Walker P. Chronic obstructive pulmonary disease. Lancet 2003; 362 (9389): 1053-61.

4. Sin DD, McAlister FA, Man SF, Anthonisen NR. Contemporary management of chronic obstructive pulmonary disease: scientific review. JAMA 2003; 290: 2301-12.

5. National Collaborating Centre for Chronic Conditions. Chronic obstructive pulmonary disease. National clinical guideline on management of chronic obstructive pulmonary disease in adults primary and secondary care. Thorax 2004; 59 Suppl 1: 1-232.

6. Fabbri LM, Hurd SS; GOLD Scientific Committee. Global Strategy for the Diagnosis, Management and Prevention of COPD: 2003 update. Eur Respir J 2003; 22: 1-2.

7. Rampulla C, Arossa W, Cerveri I. Epidemiologia e aspetti socio-econonomici in Stato dell'arte sulla BPCO. Rassegna di Patologia dell'Apparato Respiratorio 1998; 13: 460-465.

8. Maynard AM. Preparing readable patient education handouts. J Nurses Staff Dev 1999; 15: 11-18.

9. North G, Margree G, Roe M. Guidelines for producing patient information literature. Nur Stand 1996; 10: 46-48.
10. Arthur V. Written patient information: a review of the literature. J Adv Nur 1995; 21: 1081-1086.

11. Mumford ME. A descriptive study of the readability of patient information leaflets designed by nurses. $J A d v$ Nurs 1997; 26: 985-91.

12. Estrada CA, Hryniewicz MM, Higgs VB, Collins C, Byrd JC. Anticoagulant patient information material is written at high readability level. Stroke 2000; 31: 296670.

13. Joshi HB, Newns N, Stainthorpe A, et al. The development and validation of a patient-information booklet on ureteric stents. BJU Int 2001; 88: 329-34.

14. Terry L. Educational care path for the endoscopic patient. Gastroenterol Nurs 2001; 24: 34-37.

15. Walsh D, Shaw DG. The design of written information for cardiac patients: a review of the literature. J Clin Nurs 2000; 9: 658-667.

16. Winslow E. Patient education materials: can patients read them, or are they ending up in the trash? Am J Nurs 2001; 101 (10): 33-38.

17. Brock TP, Williams D, Beauchesne MF. Assessment of the readability and comprehensibility of a CFC-transition brochure. Ann Allergy Asthma Immunol 2000; 84: 211-214.

18. Leydon G, Boulton M, Moynihan C, et al. Cancer patients' information needs and information seeking behaviour: in depth interview study $B M J 2000 ; 320$ (7239): 909-9132.

19. Hermiz O, Comino E, Marks G, et al. Randomised controlled trial of home based care of patients with chronic obstructive pulmonary disease. BMJ 2002; 325 (7370): 938.

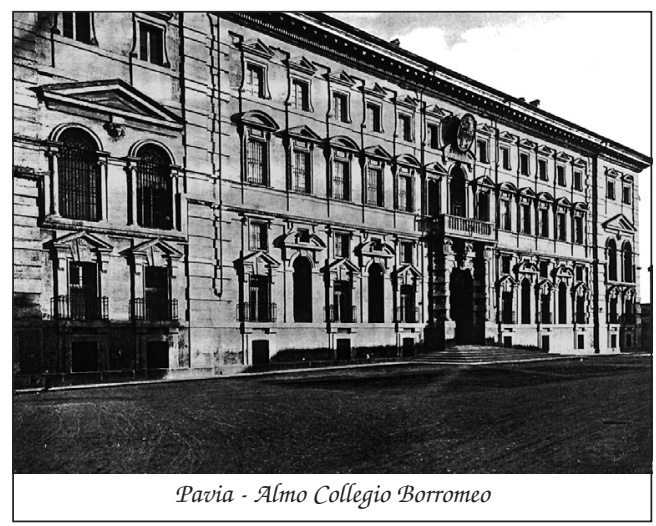

